\title{
触 New Disease Reports \\ First Report of Cherry leaf roll virus in Vaccinium darrowii
}

\author{
E.N.Y. Woo ${ }^{1 *}$, L.I. Ward ${ }^{2}$ and M.N. Pearson ${ }^{1}$ \\ ${ }^{1}$ School of Biological Sciences, The University of Auckland, Private Bag 92019, Auckland 1142, New Zealand; ${ }^{2}$ Plant Health \\ and Environment Laboratory, Ministry for Primary Industries, P.O. Box 2095, Auckland 1140, New Zealand
}

*E-mail: ewoo040@aucklanduni.ac.nz

Received: 20 Mar 2013. Published: 18 May 2013. Keywords: CLRV, blueberry, RT-PCR, phylogenetic analysis, mechanical inoculation

Cherry leaf roll virus (CLRV) is a member of the genus Nepovirus, family Secoviridae (Sanfaçon et al., 2009) and has a wide host range and geographical distribution. CLRV was first described in 1933 in Persian English walnut (Juglans regia). CLRV has subsequently been found to have a diverse host range that includes trees (American elm, ash, birch, beech), horticulturally significant species (apple, blackberry, grapevine, olive, raspberry, rhubarb, sweet cherry) and a number of shrub, weed and ornamental species. CLRV has been recorded from Europe, North America, Chile, Peru, Lebanon, Syria, Japan, New Zealand, Australia and China.

In January 2011, New Zealand blueberry bushes, belonging predominantly to two species, Vaccinium corymbosum cv. 'Ivanhoe' (Northern Highbush Blueberry) and V. darrowii cv. 'Jubilee 83' (Southern Highbush Blueberry) were surveyed for CLRV. A total of 89 leaf samples exhibiting necrotic spot lesions were collected and tested for the presence of CLRV using reverse transcription-polymerase chain reaction (RT-PCR) (Invitrogen, Carlsbad, CA, USA). Total ribonucleic acid (RNA) was extracted from leaf samples using the RNeasy ${ }^{\circledR}$ Plant Mini Kit (Qiagen, Valencia, CA, USA) as per the manufacturer's protocol. RT-PCR was conducted using the CLRV-specific primers of Werner et al. (1997). RNA extracts from all 89 leaf samples were confirmed to be RT-PCR competent using the nad5-s and nad5-as primers of Menzel et al. (2002) with a 180 bp product being obtained from all the samples tested. Five samples, all of $V$. darrowii, produced amplicons of the expected size for CLRV. The amplicons were cloned using a pGEM ${ }^{\circledR}$-T easy vector systems kit (Promega Corporation, USA) and sequenced (GenBank Accession Nos. KC753523 to KC753527). Alignment of consensus sequences showed that the five isolates shared $85.5-97.8 \%$ nucleotide identity with each other. A BLASTn search of these sequences in GenBank showed 86.8 - $99.7 \%$ nucleotide identity to four other New Zealand CLRV isolates from apple (JN581002, JQ822107) and red raspberry (AJ877162). The primer sequences described by Werner et al. (1997) were removed prior to performing a multiple sequence alignment with selected CLRV isolates from Woo et al. (2012). The phylogeny based on a maximum likelihood phylogenetic tree showed that the five blueberry isolates clustered in phylogenetic group C (Fig. 1) as designated by Rebenstorf et al. (2006). Sap from the five positive blueberry samples was used to inoculate Nicotiana occidentalis. Systemic symptoms of chlorotic spots and severe leaf deformation coupled with stunted growth and reduction of the size of leaves were observed between five to 27 days post-inoculation (Fig. 2). The presence of CLRV in N. occidentalis was confirmed by RT-PCR and

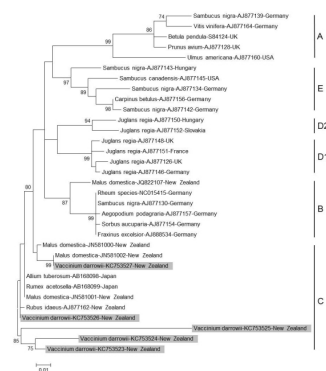

Figure 1 direct sequencing. CLRV is transmitted naturally through seed and pollen and is also transmitted mechanically and by grafting. Blueberry is propagated from seed when developing new cultivars and by cuttings for commercial production, both providing opportunities for spread of CLRV. To the best of our knowledge, this is the first report of CLRV infecting blueberry.

\section{Acknowledgements}

This study was supported by a research grant to the first author from the New Zealand Ministry for Primary Industries.

\section{References}

Menzel W, Jelkmann W, Maiss E, 2002. Detection of four apple viruses by multiplex RT-PCR assays with coamplification of plant mRNA as internal control. Journal of Virological Methods 99, 81-92. [http://dx.doi.org/10.1016/S0166-0934(01)00381-0]

Rebenstorf K, Candresse T, Dulucq MJ, Büttner C, Obermeier C, 2006. Host species-dependent population structure of a pollen-borne plant virus, Cherry leaf roll virus. Journal of Virology 80, 2453-2462.

[http://dx.doi.org/10.1128/JVI.80.5.2453-2462.2006]

Sanfaçon H, Wellink J, Le Gall O, Karasev A, van der Vlugt R, Wetzel T, 2009. Secoviridae: a proposed family of plant viruses within the order Picornavirales that combines the families Sequiviridae and Comoviridae, the unassigned genera Cheravirus and Sadwavirus, and the proposed genus Torradovirus. Archives of Virology 154, 899-907. [http://dx.doi.org/10.1007/s00705-009-0367-z ]

Tamura K, Peterson D, Peterson N, Stecher G, Nei M, Kumar S, 2011. MEGA5: Molecular evolutionary genetics analysis using maximum likelihood, evolutionary distance, and maximum parsimony methods. Molecular Biology and Evolution 28, 2731-2739.

[http://dx.doi.org/10.1093/molbev/msr121]

Werner R, Mühlbach HP, Büttner C, 1997. Detection of Cherry leaf roll nepovirus (CLRV) in birch, beech and petunia by immuno-capture RT-PCR using a conserved primer pair. European Journal of Plant Pathology 27, 309-318.

[http://dx.doi.org/10.1111/j.1439-0329.1997.tb01085.x ]

Woo ENY, Clover GRG, Pearson MN, 2012. First report of Cherry leaf roll virus (CLRV) in Malus domestica. Australasian Plant Disease Notes 7, 151-156. [http://dx.doi.org/10.1007/s13314-012-0072-8]

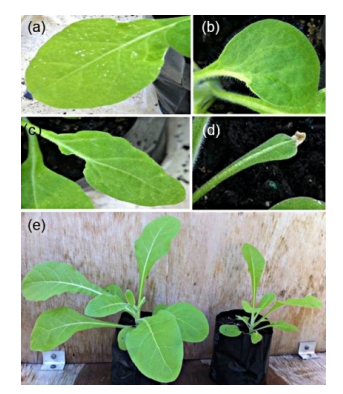

Figure 2

27. 16. [http://dx. doi. org/10.5197/j.2044-0588.2013.027.016] 